\title{
Low dose fluorescein angiography of the conjunctiva and episclera
}

\author{
PAUL A R MEYER AND PETER G WATSON \\ From the Department of Ophthalmology, Addenbrooke's Hospital, Cambridge
}

SUMMARY By reducing the dose of injected fluorescein its leakage from conjunctival and episcleral capillaries has been minimised. These vessels have been demonstrated with great clarity, and the venous circulation, previously obscured by extravascular fluorescein, has also been revealed. The anatomy of the anterior segment vessels, and the blood flow within them, has been studied in eight normal subjects. The anterior ciliary arteries feed an anterior episcleral arterial circle that has superficial and deep components. This supplies the anterior conjunctival and episcleral circulations, the limbal arcades, and the iris arterioles. Where the superficial arterial circle is deficient, isolated vessels emerge from the deep segments of the circle to supply the episcleral plexus and conjunctival arterioles. Watershed zones between the anterior and posterior territories of the conjunctival and episcleral circulations overlap. They may fluoresce up to 30 seconds after the anterior ciliary arteries. The scope of this technique and the implications of these findings are discussed.

From its introduction in 1961 fluorescein angiography rapidly became a valuable tool for examining the retinal microcirculation. ${ }^{2}$ After intravenous injection of sodium fluorescein, the retinal vessels are demonstrated in crisp contrast against a nonfluorescent background. The low molecular weight of sodium fluorescein (376.3 daltons), makes this an ideal probe with which to test the integrity of tight junctions between retinal capillary endothelial cells and between cells of the retinal pigment epithelium, as well as the permeability of Bruch's membrane.

The application of fluorescein angiography to the examination of the anterior segment of the eye was introduced for the limited purpose of demonstrating abnormalities in the iris capillaries, in which endothelial cells are joined by tight junctions. ${ }^{-26}$

However, the demonstration of episcleral and conjunctival vessels has always been a problem ${ }^{78}$ because these capillaries leak molecules smaller than serum albumin. The low molecular weight fluorescein rapidly extravasates, obliterating the angiogram, often before the venous phase has been reached. ${ }^{89}$ This restricts the diagnostic value of such angiograms to the demonstration of early leakage or

Correspondence to Dr P A R Meyer, Department of Ophthalmology, Addenbrooke's Hospital, Hills Road, Cambridge CB2 2QQ. gross hypoperfusion. ${ }^{10}$ The low molecular weight of fluorescein, of such assistance when interpreting retinal angiograms, is therefore a major obstacle during conjunctival and episcleral angiography.

If leakage is to be prevented, all the fluorescein must be associated with large molecules during its passage through the conjunctival and episcleral capillary beds. Sodium fluorescein does bind ionically to circulating albumin, though there is a wide variation between subjects, and approximately $10 \%$ remains free at steady state." This is likely to be far higher during the first pass of an injected bolus unless the fluorescein has mixed with an adequate volume of blood before reaching the target capillary bed. The time that mixing takes is directly related to the dose given. This is therefore critical, but sufficiently low doses are usually inadequate to obtain a satisfactory photographic record. However, by using sensitive photographic emulsion, and enhancing its ASA rating during development, such low doses of fluorescein can yield a good image.

Despite the presence of excellent descriptions of the circulations of the anterior segment ${ }^{12-16}$ the flow patterns within these vessels are still disputed. ${ }^{15}{ }^{17-19}$ In addition there is still no complete account of the filling and emptying of conjunctival and episcleral capillary plexuses, so vital to our understanding of episcleritis and scleritis. 


\section{Material and methods}

\section{SUBJECTS}

Eight normal subjects (six males and two females) between the ages of 20 and 35 years participated. None was aware of having suffered from any serious systemic or eye disease, and apart from the contraceptive pill none was taking any medication. All gave their informed consent.

The temporal limbus was studied by a single angiogram in every subject, and four received an additional angiogram of the nasal limbus. To demonstrate consistency of the flow pattern one volunteer underwent three identical studies of the temporal limbus.

\section{ANGIOGRAPHY TECHNIQUE}

Each study was preceded by a sequence of colour photographs of the target area (Kodak Ektachrome, 200 ASA), taken on a Zeiss photo-slit lamp at $\times 6, \times$ 10 , and $\times 16$ magnification.

The photo-slit lamp was modified for fluorescein angiography by the addition of a xenon flash unit for off-axis illumination. The excitation filter permitted a waveband of $420-495 \mathrm{~nm}$, and the T50 of the longpass barrier filter was $515 \mathrm{~nm}$. The flash generator was set to discharge 720 watts at 1.5 or 2 -second intervals. The camera was mounted on a 50:50 beam splitter, and photography was performed through an open aperture (f 14) using 400 ASA monochrome film (Kodak Tri-X). The magnification designated on the slit lamp was $\times 16$, giving a magnification factor of 3.5 at the camera back.

$0.6 \mathrm{ml}$ of $20 \%$ sodium fluorescein $(120 \mathrm{mg})$ was injected very rapidly into an antecubital vein and the photographic sequence of 30 frames began 5 seconds later.

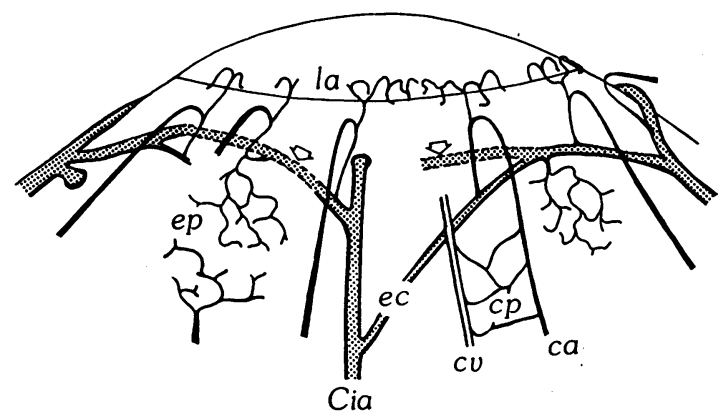

Fig. 1 Diagrammatic representation of the arterial circulations of the episclera and conjunctive: $\mathrm{Cia}=$ anterior ciliary artery. $\mathrm{ec}=$ anterior episcleral arterial circle (deep component marked $\emptyset)$. ca $=$ conjunctival arteriole. ep=episcleral capillary plexus. $\mathrm{cp}=$ conjunctival capillary plexus. la =limbal arcades. $\mathrm{cv}=$ conjunctival vein.
PHOTOGRAPHIC DEVELOPMENT

Each film was processed separately in a developing tank. The developer (Patterson Acuspeed) was diluted and filtered immediately before use. Development proceeded for 9 minutes at $20^{\circ} \mathrm{C}$, followed by conventional fixation and washing.

\section{Results}

All the angiograms clearly demonstrated the anterior ciliary vessels and the arterial, capillary, and venous phases of the conjunctival and episcleral angiograms. The peripheral iris circulation was also recorded in six of the studies.

The angiographic results were considered together in order to derive common patterns of perfusion. It is these (Fig. 1) that are presented below. With the exception of the timing of the angiographic sequence no quantification of the results was attempted.

\section{NORMAL ANTERIOR SEGMENT FLUORESCEIN ANGIOGRAM:}

\section{ARTERIAL PHASE}

The positions of the anterior ciliary arteries are inconstant as they run radially towards the limbus within Tenon's capsule. During their course over the anterior part of the globe they usually branch little, if at all.

Close to the limbus they give rise to superficial (anterior episcleral) and deep (scleral) divisions (Figs. 1, 5). The former continue in the superficial or deeper episclera. The latter penetrate the sclera and disappear from view (Fig. 5). In our subjects the scleral contributions were more prominent nasally and the episcleral divisions temporally.

Anterior episcleral arterial circle. The anterior episcleral divisions arise between 1 and $5 \mathrm{~mm}$ behind the limbus (Figs. 2, 5). They run forward and circumferentially to anastomose with contributions from the adjacent anterior ciliary arteries, so forming a fragmentary episcleral arterial circle (Fig. 2). The anastomotic vessels may be superficial (Fig. 2) or deep (Fig. 3). The fluorescence from deep vessels is hazy and indistinct (Figs. 2, 3). Superficial episcleral vessels may run deeper at their anastomoses (Fig. 3) and occasionally they dip out of view.

From the anterior episcleral arterial circle four distinct circulations are supplied: episcleral, anterior conjunctival, limbal, and iris.

Episcleral circulation. The episcleral plexus is fed anteriorly from the episcleral arterial circle (Fig. 3) and receives a posterior blood supply from vessels that appear at the level of the muscle insertions. However, areas of episcleral plexus that lie far from an anterior ciliary artery are usually perfused entirely from the episcleral arterial circle (Fig. 4). 

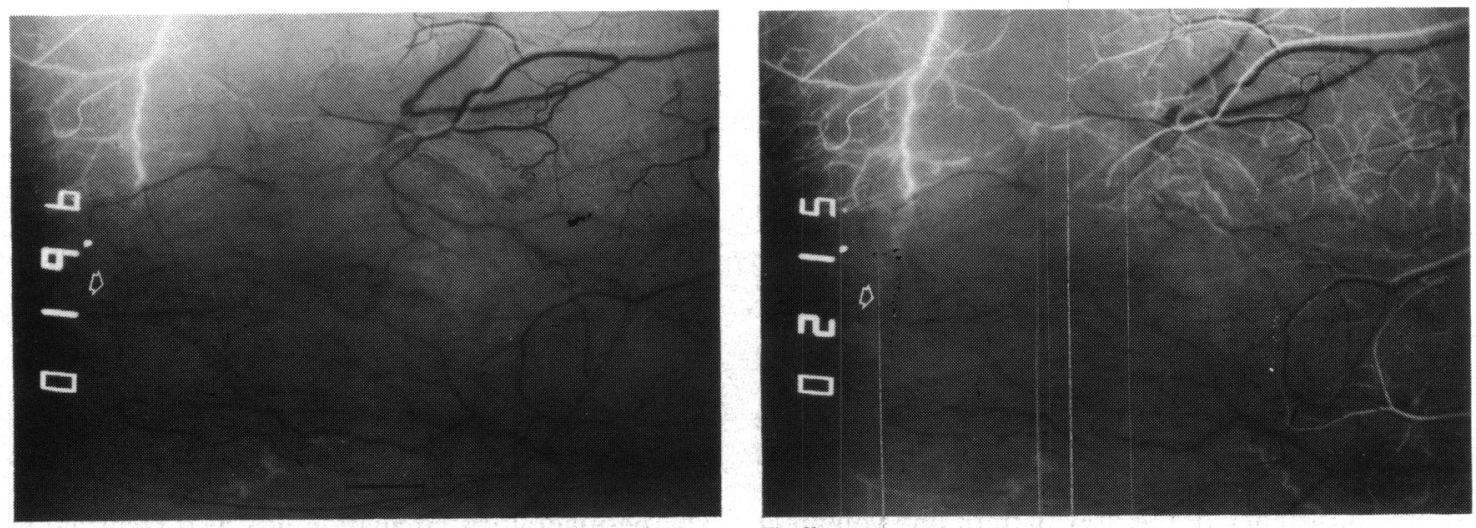

Fig. $2 \mathrm{a}$

Fig. 2b
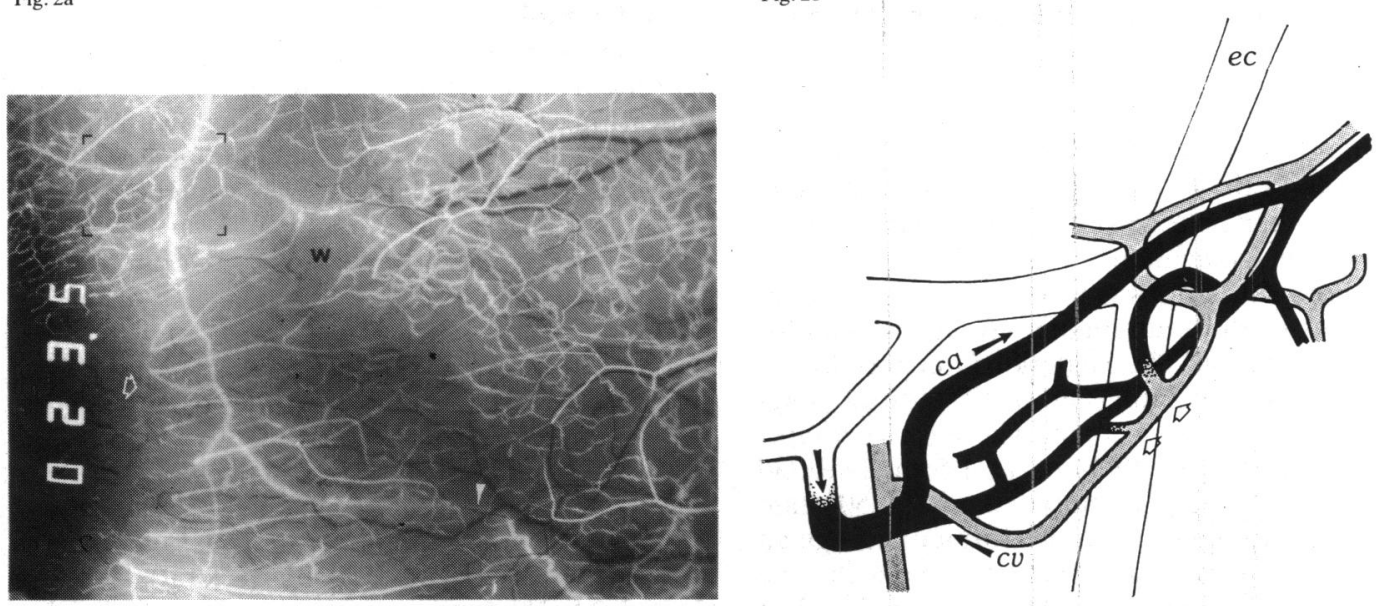

Fig. 2d

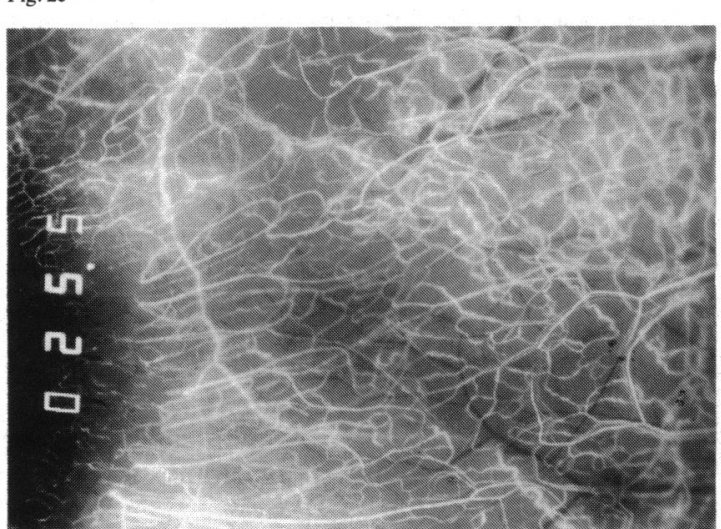

Fig. 2e

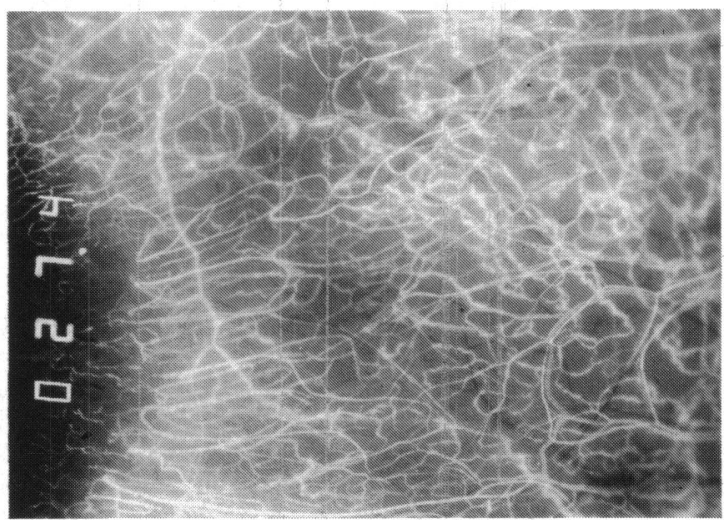

Fig. 2f

Fig. 2 Left temporal angiogram of a normal 32-year-old female. The time after injection is recorded on each frame. (Scale $=1 \mathrm{~mm})$. (2a) Fluorescein is first seen in an anterior ciliary artery branch that forms part of the anterior episcleral arterial circle. (2b) After supplying a small territory of anterior conjunctiva this vessel penetrates the sclera and continues at a deeper level ( $D$; see also Fig. 2c). The posterior tarsal circulation begins to fill. (2c) This fragment of the anterior episcleral arterial circle is completed by a superficial vessel that runs from the point of scleral penetration to an adjacent anterior ciliary artery. The vessel runs deep at ( $\nabla)$. From the episcleral arterial circle anterior roots pass forwards to the limbal reflection of the conjunctiva, where they feed the limbal arcades. They then loop back to supply a rim of anterior conjunctiva. Note the characteristic, irregular net of episcleral capillaries, over which is arrayed the lace-like conjunctival plexus. The watershed zone (W) between anterior conjunctival and posterior tarsal contributions to the conjunctival plexus lies about $3 \mathrm{~mm}$ from the limbus. This overlies the region of late perfusion of the episcleral plexus. (2d) Diagrammatic details of the area bracketed in Fig. $2 c$. Blood from the episcleral arterial circle (ec) is distributed to an anterior root, and thence into conjunctival arterioles (ca). Shunting of blood $(\Delta)$ from an arteriole into an adjacent conjunctival venule (cv) explains the early perfusion of anterior conjunctival venules and the delayed fuorescence of the watershed zone $(\mathrm{W}) . \mathrm{ec}=$ anterior episcleral arterial circle. $\mathrm{ca}=$ conjunctival arteriole. $\mathrm{cv}=$ conjunctival venule. $(2 \mathrm{e}, \mathrm{f})$ Many radial anterior conjunctival vessels are veins and in the posterior tarsal circulation veins accompany arteries. Leakage of fluorescein is minimal and never obscures anatomical detail. 
The small vessels that supply the episcleral plexus may arise as posterior branches from either the superficial or the deep contributions to the anterior episcleral arterial circle (Fig. 3). Where neither a superficial nor a deep component of the episcleral arterial circle can be seen, the episcleral plexus fills slowly from isolated vessels that emerge from the sclera close to the limbus (Fig. 4). These fluoresce very shortly after the visible episcleral circle. They are identical to episcleral branches from the deeper sections of the visible episcleral arterial circle that can be found in other subjects (Fig. 3).

Anterior conjunctival arterioles. Throughout their superficial course the arteries composing the episcleral circle give rise to fine loops that run forwards into the limbal reflection of the conjunctiva before curving back radially to feed the lacework of the anterior conjunctival capillary plexus (Fig. 2). The delicate column of blood within the anterior conjunctival loops may be punctuated by a string of individual erythrocytes, suggesting that the lumen is in the order of $12 \mu \mathrm{m}$ (Figs. 2, 5, 6).

Anterior conjunctival loops may also arise from arterioles that emerge from the sclera (Fig. 5).

The posterior conjunctival circulation, which is derived from the posterior tarsal vessels, fills after the anterior conjunctival circulation temporally, but this sequence is often reversed nasally (Fig. 5). Anterior conjunctival loops sometimes anastomose with arterioles of similar calibre derived from the posterior tarsal circulation (Fig. 6).
Limbal arcades (Figs. 5, 6). These are supplied by anterior branches from the episcleral circle, and their origins are usually shared with those of the anterior conjunctival loops. They may fill very late during a normal angiogram.

Iris vessels. The first flush of fluorescein within the radial arterioles of the iris usually coincides with filling of the anterior episcleral arterial circle (Figs. $3,4)$.

\section{CA PILLARY PHASE}

Episclera. The episcleral capillaries, though always visible, may be difficult to discern below the more prominent conjunctival circulation. They are most clearly seen when the conjunctival circulation fills late for anatomical or pathological reasons (Figs. 2 , 4). Fluorescence slowly spreads between the posterior branches of the episcleral circle to illuminate a delicate capillary network covering the sclera. This net also fills from behind, and the anterior and posterior territories meet at a variable distance behind the limbus - a boundary that can normally be recorded (Fig. 2).

Conjunctiva. Fine capillaries form a web that joins the parallel anterior conjunctival arterioles and venules (Figs. 1,2). Capillaries closest to the limbus fill first and shunt fluorescein into conjunctival venules before any reaches the distal capillary bed (Fig. 2d). The watershed zone separating the territories supplied by the anterior ciliary and posterior tarsal systems may fluoresce very late.

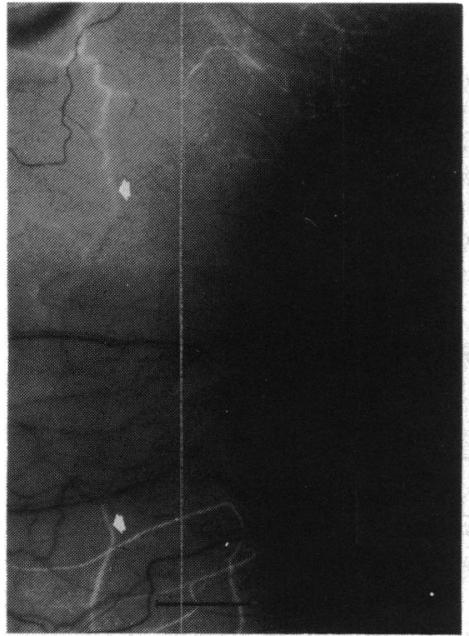

Fig. 3a

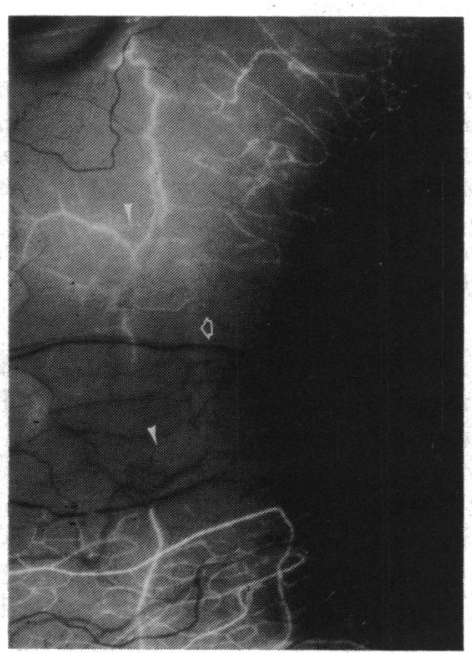

Fig. 3b

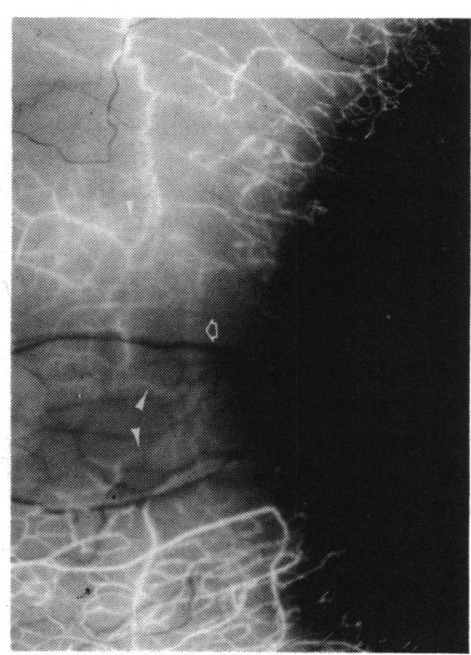

Fig. 3c

Fig. 3 Right temporal angiogram of a normal 23-year-old female. a: First flush. b-c: Two and four seconds later. (Scale $=1$ $\mathrm{mm})$. Demonstration of the superficial $(\$)$ and deep $(\diamond)$ components of the anterior episcleral arterial circle. The episcleral plexus fills from both the superficial and the deep parts of the circle $(\nabla)$. Note the radial iris arterioles. 


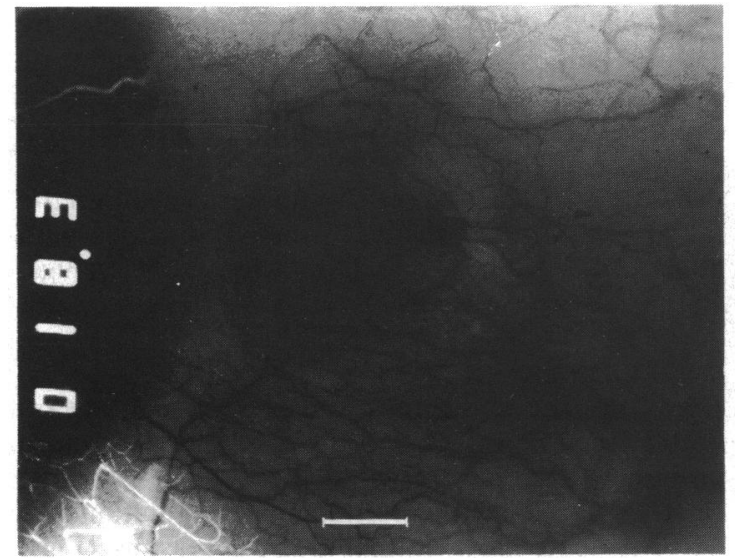

Fig. 4a

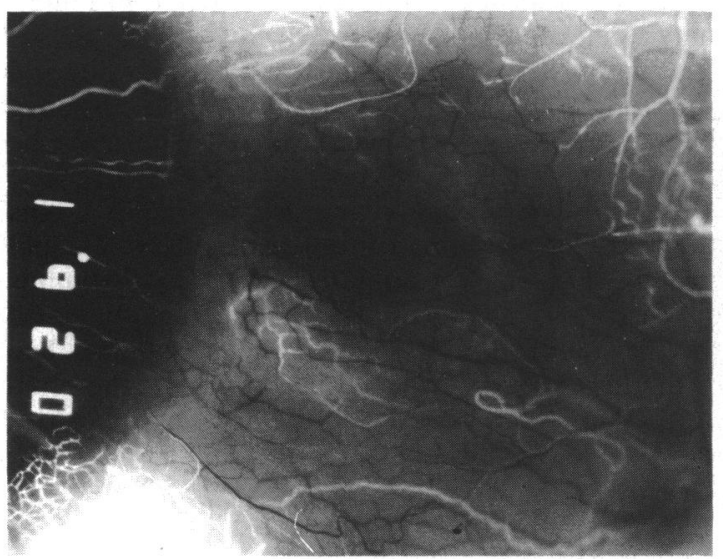

Fig. 4c

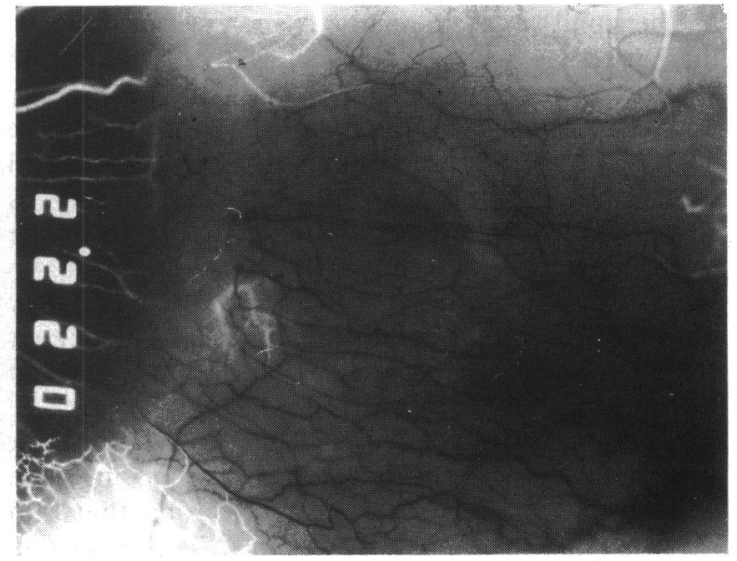

Fig 4b

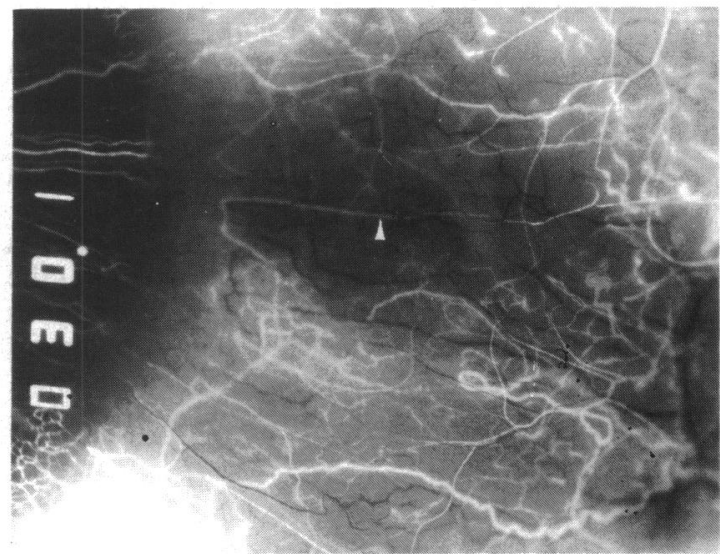

Fig 4d

Fig. 4 Left temporal angiogram of a normal 34-year-old male. Times after injection recorded on film: first flush was at $16 \cdot 4 \mathrm{~s}$. $($ Scale $=1 \mathrm{~mm})$. The anterior conjunctival and limbal circulations adjacent to an anterior ciliary artery trunk fill early. The deep component of the anterior episcleral arterial circle and the iris arterioles fluoresce simultaneously. A large expanse of sclera and conjunctiva that is distant from any anterior ciliary artery remains non-perfused until late into the angiogram. The episcleral net in this region is slowly filled from a vessel that emerges from the sclera. This vessel presumably arises from the deep component of the episcleral arterial circle (cf. Fig. 3). An episcleral vein ( $\mathbf{\Delta})$ passes deep to the silhouettes of the anterior conjunctival vessels.which are still non-perfused 14 seconds after first flush.

\section{VENOUS PHASE}

Centripetal venules drain the episcleral plexus towards the limbus (Fig. 4) and venules accompany the anterior conjunctival arterial loops (Fig. 6) or are interspersed between them (Fig. 2). Although the anterior episclera and conjunctiva drained towards the limbus (Fig. 4), this study revealed no well organised superficial venous ring corresponding to the anterior episcleral arterial circle.

The posterior conjunctiva drains back towards the tarsal circulation (Fig. 2).

The large episcleral vessels, which so often appear to pass no blood in conventional angiograms, were frequently seen to carry fluorescein during the late venous phase (Fig. 5).

TIMING

An angiographic sequence began with the appearance of fluorescein in the anterior ciliary arteries (Fig. 5). The filling of iris arterioles either coincided or followed within 2 seconds.

Filling of the anterior episcleral arterial circle took between 1.5 and 14 seconds. It is a variable anatomical entity and the interpretation of its limits inevitably influenced the delay that was recorded.

Anterior conjunctival arterioles fluoresced within 


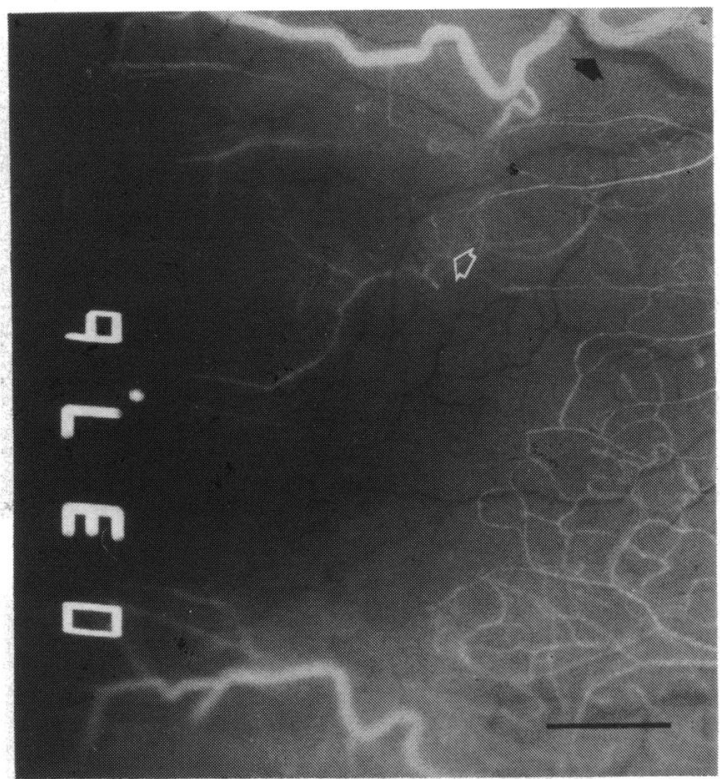

Fig. 5a

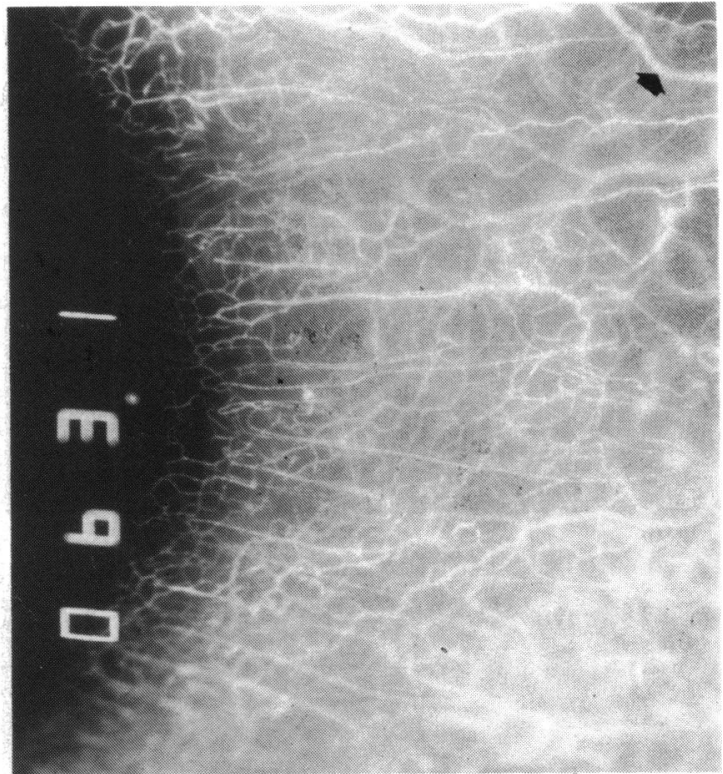

Fig. 5b

Fig. 5 Right nasal angiogram of a normal 26-year-old male. (Scale $=1 \mathrm{~mm})$. a (6 seconds after first flush): Conjunctival filling from the posterior tarsal circulation precedes perfusion from the limbus. The large vessels that fill early are anterior ciliary artery trunks. Their divisions may penetrate the sclera, continue in the superficial episclera, or follow an intermediate course. One such vessel re-emerges from the sclera at $(\downarrow)$, where it contributes to the episcleral plexus and gives rise to a conjunctival arteriole. Note the large, non-perfused episcleral vessel $(\$) . \mathrm{b}(31.5$ seconds after first flush): There is complete filling of the limbal arcades, which do not leak. The deep vascular silhouette (\$) now fluoresces during the late venous phase.

1.5 seconds after the segment of the anterior episcleral arterial circle that supplied them. Over the temporal globe this preceded posterior tarsal fluorescence by up to 4 seconds.

The times taken to fill the capillary plexuses are deceptive, since the episcleral plexus cannot be seen in its entirety, and the focusing of the angiogram may affect the definition of the limbal arcades. The most useful generalisations were that: all the capillaries seen in the angiogram were perfused between 6 and 30 seconds after first flush; the limbal arcades and the episcleral net filled most slowly.

Fluorescein sometimes appeared in conjunctival venules within 4 seconds of first flush and long before full perfusion of the capillary bed (see above). This made the distinction of venules from arterioles extremely difficult in some angiograms. Veins fluoresced correspondingly early (between 4 and 10 seconds after first flush), the first to do so being those draining the anterior conjunctiva.

The large episcleral veins usually filled late in the angiogram.

\section{LEAKAGE}

Leakage was first seen at between 4 and 10 seconds and its quantity varied widely. It was confined to capillaries of the conjunctiva and episclera (Fig. 6), and the walls of arteries and veins never stained. The limbal arcades did not leak for at least 30 seconds (Fig. 6), but free fluorescein entered the limbal zone at between 60 to 120 seconds, probably from the surrounding conjunctiva. The cornea never fluoresced.

There was minimal masking of anatomical detail before 30 seconds after first flush. When low dose anterior segment angiograms were repeated after an interval of 24 hours, insignificant background fluorescence persisted.

\section{Discussion}

By the simplest of modifications the technique of anterior segment fluorescein angiography has been considerably refined. Without interfering with the observation of arteries, the reduction in fluorescein dosage permits a reliable demonstration of the full sequence of capillary filling. Furthermore, the close association of fluorescein with albumin means that its immediate leakage always implies vascular pathology. The pattern of episcleral and conjunctival 


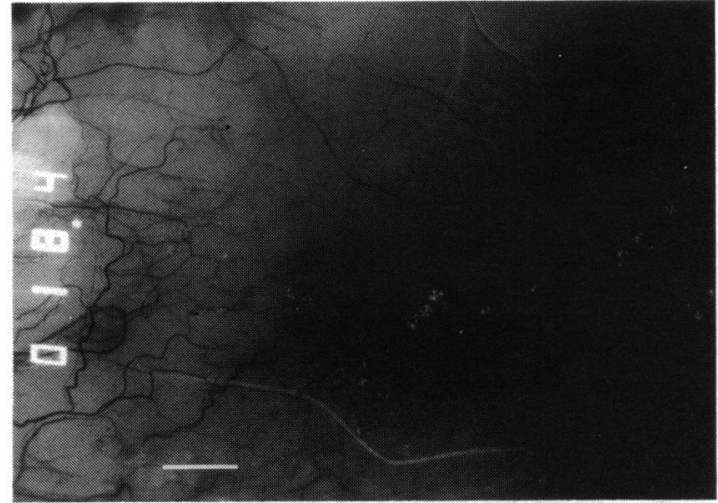

Fig. $6 a$

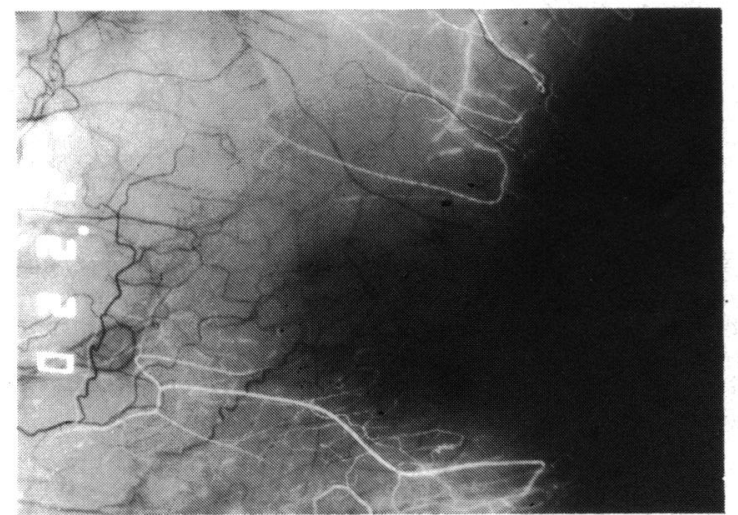

Fig. 6c

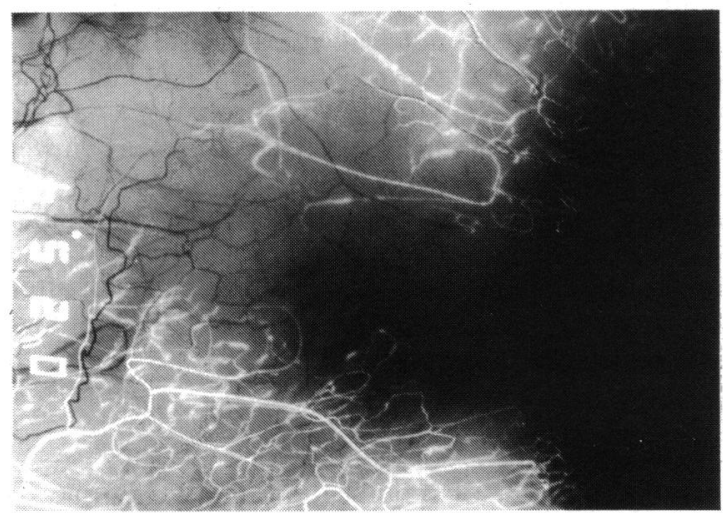

Fig. 6e

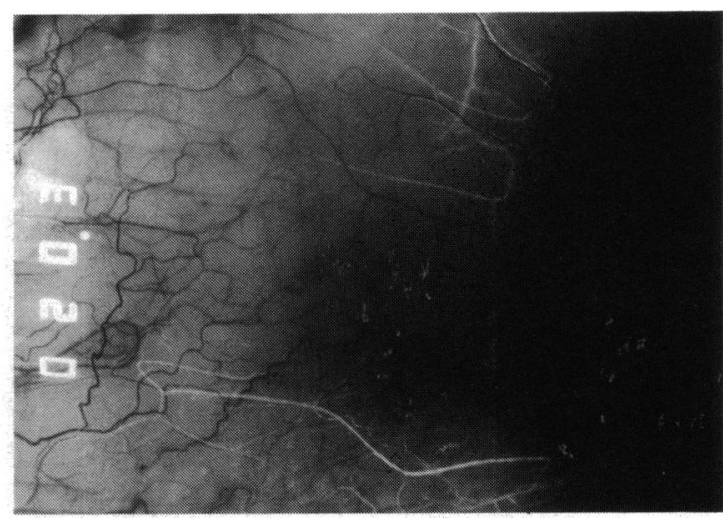

Fig. 6b

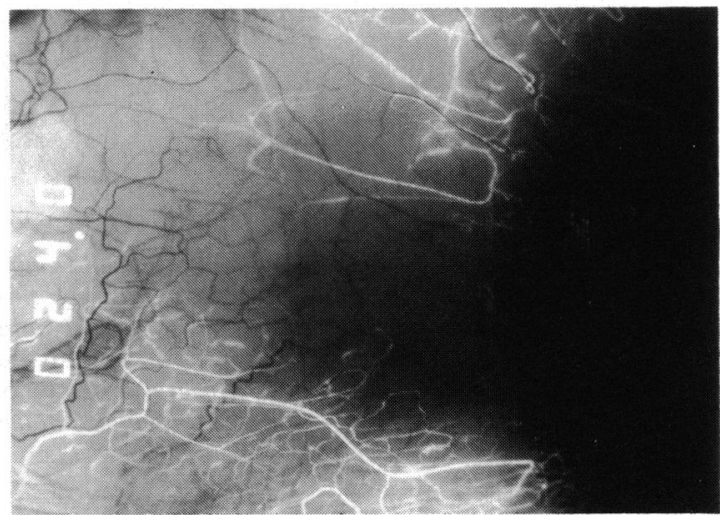

Fig. 6d

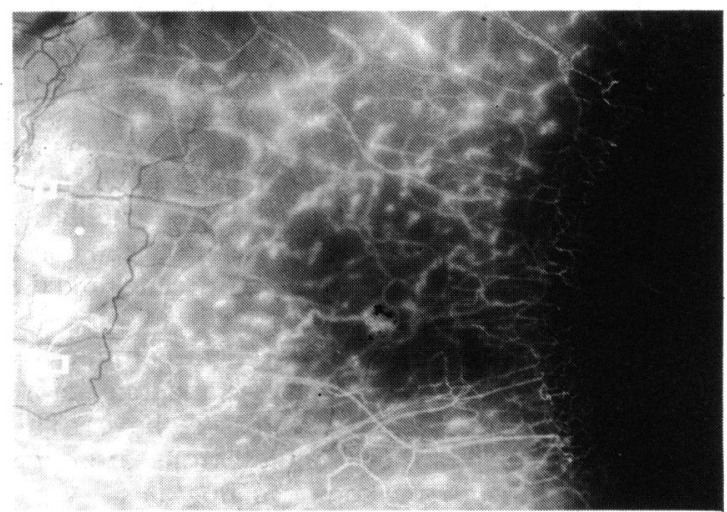

Fig. 6f

Fig. 6 Right temporal angiogram of a normal 35-year-old male. Angiographic sequence from first flush: times recorded on film. (Scale $=1 \mathrm{~mm})$. Note the anastomosis between anterior ciliary and posterior tarsal circulations. An area of episclera far from any anterior ciliary artery trunk is perfused late in the angiogram from the adjacent episcleral plexus. The limbal arcades fill completely, 20 seconds after first flush and they do not leak fluorescein. Anterior conjunctival venules accompany the arterioles. Leakage of fluorescein from anterior conjunctival and episcleral capillaries begins at 4 seconds and is particularly brisk in this angiogram. However, there is still minimal masking of anatomical detail during the first 30 seconds. 
perfusion has been clarified and the venous circulation, hitherto obscured by early leakage, has now been revealed.

The technique that is outlined above was arrived at after experiments in which rabbits received low doses of fluorescein conjugates of dextran (molecular weight 70000 ) intravenously. Leakage was avoided and high quality angiograms were obtained after the injection of as little as $5 \mu \mathrm{g}$ fluorescein isothiocyanate per $\mathrm{kg}$ body weight (Meyer $\mathrm{P}$, in preparation).

It is not only the photographic techniques that make such a reduction in dosage possible. Injected fluorescein isothiocyanate (FITC)-dextran conjugates remain within the intravascular compartment elsewhere in the body,${ }^{20}$ increasing the dosage to the eye. Furthermore the absence of leakage from anterior segment vessels heightens the contrast of the image. When FITC conjugates are approved for clinical use, FITC-dextran or FITC-human serum albumin will become the fluorescent probes of choice for anterior segment angiography. Until then, by restriction of the dose of sodium fluorescein to permit early and near-total ionic binding to serum albumin, we can approach this ideal state of affairs.

Although the partitioning of fluorescein between its free and bound forms strongly favours its association with albumin, approximately $10 \%$ of fluorophore remains free, ${ }^{11}$ and that did inevitably leak from conjunctival capillaries. This was undetectable before 4 seconds, and never interfered with the interpretation of microvascular anatomy. Nevertheless, the dual problems of leakage and anatomical variation still preclude reliable estimation of relative flow rates in the anterior segment.

Because of its considerable variability, the circulation of the conjunctiva and episclera gives a random appearance, which anatomists have long attempted to clarify. ${ }^{1215} 16$ Despite differences between mammalian species, Morrison and Van Buskirk's elegant methacrylate casts of anterior segment vessels in the monkey are a useful starting point. ${ }^{21}$

The monkey anterior segment is perfused from an anterior episcleral arterial circle, fed by the anterior ciliary arteries. We consider its human counterpart, although asymmetrical and incomplete, to be equally crucial. Our angiograms show that it too arises from the anterior ciliary arteries, supplies the anterior conjunctival, anterior episcleral, and limbal circulations, and may also feed the iris arterioles. This concords with the anatomical descriptions of Leber ${ }^{12}$ and Ashton and Smith. ${ }^{15}$

The episcleral arterial circle broadly resolves into superficial and deep components. This raises the possibility that its deeper component is the 'incomplete arterial circle' that Ashton and Smith described in association with the canal of Schlemm. ${ }^{15}$
The relationship between the episcleral arterial circle and the major circle of the iris cannot be resolved angiographically at present. Their simultaneous perfusion leaves no reason to doubt that they share a blood supply, but the relative contributions from the anterior and long posterior ciliary arteries must remain a matter of conjecture.

The anterior episcleral capillary bed is filled centrifugally by fine branches from the superficial or deep components of the episcleral arterial circle. However, these vessels feed only a narrow rim of episclera around the limbus. Beyond this the episcleral net is supplied by vessels that appear at the level of the rectus muscle insertions-probably branches of the anterior ciliary arteries. Perfusion of the zone separating these territories is tenuous, and the very frequent occurrence of scleritis in this region emphasises the importance of ischaemia to its aetiology.

The posterior limits of the territories of anterior conjunctiva and of episclera supplied by the episcleral arterial circle are superimposed. Closure of this common blood supply may explain why conjunctival infarction often overlies areas of severe scleritis.

The limbal arcades were completely perfused in all our studies, in contrast to the observations of Mitsui et al. ${ }^{7}$ Vessels that filled late during their study were probably lost in the sea of background fluorescence. Until fluorescein had permeated the subconjunctival space, we found none in the limbal perivascular compartment. It may enter this region not by leakage from the arcades, but by diffusion from adjacent conjunctival capillaries.

The paradoxical paucity of anterior conjunctival venules in angiograms, despite their apparent profusion during slit-lamp examinations, ${ }^{8}$ is simply explained once these vessels can be examined with adequate resolution. It transpires that the conjunctival capillary bed is arrayed in parallel between the radial arterioles and venules. Proximal capillaries fill first and shunt blood into conjunctival venules. Consequently, venous drainage close to the limbus precedes the arterial phase in the more distal conjunctiva: many of the radial conjunctival vessels that fill early are in fact venules. Blood flow through the distal anterior conjunctival circulation may be controlled by variations in the resistance within proximal capillaries.

Large vessels in which no flow can be recorded, previously considered to be a feature of the normal circulation, ${ }^{9}$ were observed in the earlier frames of our angiograms. Bron and Easty ${ }^{8}$ thought that they carried diluted fluorescein and became lost amidst the high level of background fluorescence that has hitherto plagued anterior segment angiography. 
We can confirm this, since most of these vascular silhouettes did ultimately fill in our angiograms, and proved to be large veins.

The direction of flow within the anterior ciliary arteries is disputed. Some authors ${ }^{919}$ have equated them with the 'emissary veins' described by Kiss in $1943,{ }^{17}$ in which blood appears to flow away from the limbus. ${ }^{18}$ However, published angiographic sequences in which fluorescence of anterior ciliary arteries progresses centrifugally ${ }^{1019}$ can also be explained by poor illumination of the peripheral photographic field. Since the rate of flow in arterioles of $100 \mu \mathrm{m}$ diameter is approximately $20 \mathrm{~mm} / \mathrm{s},{ }^{22}$ the resolution of this controversy is beyond the scope of this study, in which the minimum time interval between photographs was 1.5 seconds.

Although the filling sequence of the angiogram is reliably reproducible at any one site in a single individual, the timing varies enormously. There is also vast variation between subjects. This means that, even if a previous angiogram of the same area is available, the time taken for capillary beds to fill cannot be usefully compared.

Delayed perfusion has for some time been considered to be a hallmark of necrotising scleritis. ${ }^{10}$ While this is true, the critical watershed zones can take 30 seconds to fill in normal people. Since capillary detail is lost by that time in many full-dose angiograms, they must be interpreted with great caution.

The low dose of fluorescein that we administer should reduce the morbidity of fluorescein angiography. Indeed no adverse effects have yet been recorded during any of the 30 angiograms that we have so far performed.

Capillary beds that usually leak fluorescein may now be examined by fluorescein angiography. This paper offers an introduction to the angiographic appearances of the anterior segment of the normal eye, but the method is equally applicable to the observation of circulations exposed at surgery or endoscopy.

We thank Mr K Harvey for his help with the development of the photographic techniques and Miss C Barber for photographing the angiograms.

Paul Meyer is in receipt of a research fellowship from the Wellcome Trust.

\section{References}

1 Maclean A, Maumenee AE. Haemangioma of the choroid. Am J Ophthalmol 1960; 50: 3-11.

2 Novotny HR, Alvis DL. A method of photographing fluorescence in circulating blood in the human retina. Circulation 1961; 24: $82-6$.

3 Cobb B. Vascular tufts at the pupillary margin. Trans Ophthalmol Soc UK 1968; 88: 211-21.

4 Jensen VA, Lundback K. Fluorescence angiography of the iris in recent and long-term diabetes: preliminary communication. (XVII Scandinavian Ophthalmological Congress, Copenhagen 1967.) Acta Ophthalmol (Kbh) 1968; 46: 584-5.

5 Rosen ES, Lyons D. Microhaemangiomata at the pupillary borders demonstrated by fluorescence photography. Am J Ophthalmol 1969; 67: 846-53.

6 Brunn-Jensen J. Fluorescein angiography of the anterior segment. Am J Ophthalmol 1969; 67: 842-5.

7 Mitsui Y, Matsubara M, Kanawaga M. Fluorescence iridocorneal photography. Br J Ophthalmol 1969; 53: 505-12.

8 Bron AJ, Easty DL. Fluorescein angiography of the globe and anterior segment. Trans Ophthalmol Soc UK 1970; 90; 339-67.

9 Marsh RJ, Ford SM. Blood flow in the anterior segment of the eye. Trans Ophthalmol Soc UK 1980; 100: 388-99.

10 Watson PG, Bovey E. Anterior segment fluorescein angiography in the diagnosis of scleral inflamation. Ophthalmology (Rochester) 1985; 92: 1-11.

11 Palestine AG, Brubaker RF. Plasma binding of fluorescein in normal subjects and in diabetic patients. Arch Ophthalmol 1982; 100: $1160-1$.

12 Leber T. Die Cirkulations- und Ernahrungsverhältnisse des Auges. Graefe-Saemisch Handbuch der Gesamten Augenheilkunde. Leipzig: Engelmann, 1903: 2: chapter 1.

13 Ashton N. Anatomical study of Schlemm's canal and aqueous veins by means of Neoprene casts. Br J Ophthalmol 1951; 35: 291-303.

14 Ashton N. Anatomical study of Schlemm's canal and aqueous veins by means of Neoprene casts. Part II: aqueous veins. $\mathrm{Br} J$ Ophthalmol 1952; 36: 265-7.

15 Ashton N, Smith R. Anatomical study of Schlemm's canal and aqueous veins by means of Neoprene casts. Part III: arterial relations of Schlemm's canal. Br J Ophthalmol 1953; 37: 577-86.

16 Duke-Elder S, Wybar KC. Blood vessels and nerves of the eye. In: Duke-Elder S, ed. System of ophthalmology. London: Kimpton, 1961; 2: chapter 4.

17 Kiss F. Der Blutkreislauf des Auges. Ophthalmologica 1943; 106: $225-50$.

18 Weinstein P. New concepts regarding anterior drainage of the eye. Br J Ophthalmol 1950; 34: 161-8.

19 Talusan ED, Schwartz B. Fluorescein angiography: demonstration of flow pattern in anterior ciliary arteries. Arch Ophthalmol 1981; 99: 1074-80.

20 Arfors KE, Hint HC. Studies of the microcirculation using fluorescent dextran. Microvasc Res 1970; 3: 440 (abstr).

21 Morrison JC, Van Buskirk EM. Anterior collateral circulation in the primate eye. Ophthalmology 1983; 90: 707-15.

22 Tanaka T, Riva C, Ben-Sira I. Blood velocity measurements in human retinal vessels. Science 1974; 186: 830-1.

Accepted for publication 25 March 1986. 